\title{
ORTHOGONAL DECOMPOSITION OF ISOMETRIES IN A BANACH SPACE
}

\author{
G. D. FAULKNER AND J. E. HUNEYCUTT, JR.
}

\begin{abstract}
In this paper the Wold decomposition theorem is proved for a class of isometries in smooth reflexive Banach spaces. The class in particular contains all isometries of $L^{p}(\mu)$ spaces for arbitrary measures $\mu$.
\end{abstract}

It is well known that an isometry from a Hilbert space $H$ into itself can be represented as the direct sum of two isometries, one being unitary (invertible) and the other being a shift. It is the purpose of this paper to investigate conditions which ensure that an isometry on a Banach space be represented in a similar fashion.

Let $X$ be a Banach space. For $x, y \in X$ we shall say that $x$ is orthogonal to $y(x \perp y)$ if for each $\alpha \in \mathbf{C},\|x\| \leqslant\|x+\alpha y\|$. We note that this is a nonsymmetric notion of orthogonality but that it is equivalent to the usual concept of orthogonality in Hilbert space. We write $M \perp N$ in case $M, N \subseteq X$ and $x \in M, y \in N \Rightarrow x \perp y$.

Definition. A semi-inner-product (s.i.p.) on $X$ is a function $[\cdot, \cdot]$ from $X \times X$ into $\mathbf{C}$ with the following properties:

(1) $[\cdot, y]$ is linear for each $y \in X$,

(2) $\mid[x, y] \leqslant\|x\| \cdot\|y\|$,

(3) $[x, x]=\|x\|^{2}$ for each $x \in X$,

(4) $[x, \alpha y]=\bar{\alpha}[x, y]$ for each $x, y \in X$ and $\alpha \in \mathbf{C}$.

General facts concerning semi-inner-products may be found in [2], [5]. For $M, N \subseteq X$, we write $[M, N]$ for the collection of numbers of the form $[x, y]$ where $x \in M$ and $y \in N$. It is natural to define orthogonality in terms of the s.i.p., but a particular Banach space may have many s.i.p.'s consistent with the norm and the notions of orthogonality will be dependent on the s.i.p. However, we do have the following result:

THEOREM [1]. Let $X$ be a normed linear space and $M$ and $N$ be subspaces of $X$ with $M \perp N$, then there is a s.i.p. $[\cdot, \cdot]$ such that $[N, M]=\{0\}$.

In the present paper we shall be concerned with orthogonal complements and so we have the following two lemmas concerning such complements.

LEMMA 1. Let $X=M \oplus N$ where $M$ and $N$ are subspaces of $X$ with $M \perp N$. Then $N=\{x \in X:[x, M]=0\}$ for some s.i.p. $[\cdot, \cdot]$.

Presented to the Society, March 31, 1977; received by the editors March 4, 1977. AMS (MOS) subject classifications (1970). Primary 47A65.

C) American Mathematical Society 1978 
Proof. Choose a s.i.p. such that $[N, M]=0$. Then if $[x, M]=0$, express $x$ as $x=m+n$ with $m \in M, n \in N$, then

so $x=n \in N$.

$$
0=[x, m]=[m+n, m]=\|m\|^{2}
$$

LEMMA 2. Let $M$ and $N$ be closed subspaces of $X$ with $M \perp N$, then $M \oplus N$ is closed.

Proof. Again choose a s.i.p. such that $[N, M]=0$. Let $z_{n}=x_{n}+y_{n}$ where $x_{n} \in M$ and $y_{n} \in N$; if $z_{n} \rightarrow z \in X$, then

$$
\begin{aligned}
\left\|z_{n}-z_{m}\right\| \cdot\left\|x_{n}-x_{m}\right\| & \geqslant\left|\left[z_{n}-z_{m}, x_{n}-x_{m}\right]\right| \\
& =\left|\left[x_{n}+y_{n}-x_{m}-y_{m}, x_{n}-x_{m}\right]\right| \\
& =\left|\left[x_{n}-x_{m}, x_{n}-x_{m}\right]+\left[y_{n}-y_{m}, x_{n}-x_{m}\right]\right| \\
& =\left\|x_{n}-x_{m}\right\|^{2} .
\end{aligned}
$$

Thus $\left\|x_{n}-x_{m}\right\| \leqslant\left\|z_{n}-z_{m}\right\|$, so $\left\{x_{n}\right\}$ must be Cauchy and hence $x_{n} \rightarrow x$ for some $x \in M$. It follows that $y_{n} \rightarrow z-x$ and $z-x=y \in N$. Therefore $z_{n} \rightarrow x+y \in M \oplus N$ so $M \oplus N$ is closed.

In a smooth Banach space, the s.i.p. is unique so we may write $M^{\perp}$ to stand for $\{x \mid[x, M]=0\}$.

LEMMA 3. Let $X$ be a smooth, reflexive Banach space and suppose that $\left\{M_{k}\right\}$ and $\left\{N_{k}\right\}$ are sequences of closed subspaces such that

(1) $X=M_{k} \oplus N_{k}$,

(2) $N_{k} \perp M_{k}$,

(3) $N_{k} \subseteq N_{k-1}$ and $M_{k} \supseteq M_{k-1}$.

Let $N=\cap_{k=1}^{\infty} N_{k}$ and $M=\overline{\left[\cup_{k=1}^{\infty} M_{k}\right]}$; then $X=M \oplus N$ and $N \perp M$.

Proof. Suppose $z=n_{k}+m_{k}$ where $n_{k} \in N_{k}$ and $m_{k} \in M_{k}$ for each natural number $k$; then

$$
\|z\|=\left\|n_{k}+m_{k}\right\| \geqslant\left\|n_{k}\right\| \quad(\text { by }(2)) .
$$

Thus $\left\{n_{k}\right\}$ is a bounded sequence in $X$ and hence has a weakly convergent subsequence $\left\{n_{k_{i}}\right\}$. If $n_{k_{i}}$ converges weakly to $n$, then $m_{k}=z-n_{k}$ converges weakly also, say to $m=z-n$. Now $n \in \cap_{k=1}^{\infty} N_{k}$ and $m \in \overline{\left[\cup_{k=1}^{\infty} M_{k}\right]}$. Thus $X=M+N$. If $n \in N$ and $m \in \cup M_{k}$, then $[m, n]=0$. Since $[\cdot, n]$ is continuous, we have that if $n \in N$ and $m \in M$, then $[m, n]=0$, so $N \perp M$. If $m \in N \cap M$, then $[m, m]=0$ so $m=0$; thus $X=M \oplus N$.

DEFINITION. Let $V$ be an isometry on the normed linear space $X . V$ is said to be orthogonally complemented provided that there exists a closed subspace $M$ of $X$ such that $X=M \oplus V(X)$ and $V(X) \perp M$.

We note that $V$ is orthogonally complemented if and only if there exists a projection $P: X \rightarrow V(X)$ of norm 1 .

In a Hilbert space, each isometry is orthogonally complemented. If 
$(\Omega, \Sigma, \mu)$ is a $\sigma$-finite measure space, then for $p \neq 2(1 \leqslant p<\infty)$, each isometry $V: L^{p}(\Omega) \rightarrow L^{p}(\Omega)$ is of the form $V f(x)=h(x) T f(x)$ where $T$ is a regular set isomorphism of $\Sigma$ and $|h|^{p}=d \nu / d \mu$ for $\nu(A)=\mu\left(T^{-1} A\right)$ [4]. (Here $T f$ is defined by extending to all of $L^{p}(\Omega)$, the mapping $T\left(1_{A}\right)=1_{T A}$.) Define $g$ by

$$
g(x)=\frac{1}{h(x)} 1_{\{h \neq 0\}} .
$$

If $g \in L^{p}(\Omega)$, then $V$ is orthogonally complemented. In particular, $T$ is measure preserving if and only if $|h|=1$ a.e.; so every classical shift for $L^{p}(\Omega)$ is orthogonally complemented (as is every isometry in $l_{p}$ ). ${ }^{1}$ A similar condition can be given for isometries in certain Orlicz spaces [6].

Definition. An isometry $V: X \rightarrow X$ will be called a unilateral shift if there exists a subspace $L \subseteq X$ such that

(1) for $n \geqslant m, V^{n}(L) \perp V^{m}(L)$,

(2) $X=\bigoplus_{n=0}^{\infty} V^{n}(L)$.

The following theorem generalizes a result of Wold for isometries on Hilbert space.

THEOREM. Let $V$ be an isometry on the smooth, reflexive Banach space $X$. If $V$ is orthogonally complemented then there exist closed subspaces $X_{1}$ and $X_{2}$ such that

(1) $X_{1}$ and $X_{2}$ are invariant under $V$,

(2) $\left.V\right|_{X_{1}}$ is unitary (surjective),

(3) $\left.V\right|_{X_{2}}$ is a unilateral shift,

(4) $X=X_{1} \oplus X_{2}$.

Proof. Let $L=V(X)^{\perp}$, then by hypothesis, $X=V(X) \oplus L$. By [3] we know that $[V x, V y]=[x, y]$ so for $n \geqslant m, V^{n}(L) \perp V^{m}(L)$. Since $X$ is smooth,

$$
V^{n}(L) \perp \bigoplus_{k=0}^{n-1} V^{k}(L)
$$

so by a previous lemma, the subspace $L_{n}=\bigoplus_{k=0}^{n} V^{k}(L)$ is closed for each $n$. Since $X=V(X) \oplus L$, then $V(X)=V^{2}(X) \oplus V(L)$; so

$$
X=V^{2}(X) \oplus V(L) \oplus L=V^{2}(X) \oplus L_{1} \quad \text { and } \quad V^{2}(X) \perp L_{1} .
$$

In general, $X=V^{n}(X) \oplus L_{n-1}$ and $V^{n}(X) \perp L_{n-1}$. The sequence $\left\{V^{n}(X)\right\}$ decreases and the sequence $\left\{L_{n}\right\}$ increases so we set $X_{1}=\bigcap_{n=0}^{\infty} V^{n}(X)$ and

$$
X_{2}=\overline{\bigcup_{n=0}^{\infty} L_{n}}=\bigoplus_{n=0}^{\infty} V^{n}(L) .
$$

Then by Lemma 3, $X=X_{1} \oplus X_{2}$, and $X_{1}$ and $X_{2}$ are invariant under $V$. By construction, $\left.V\right|_{X_{2}}$ is a shift and $V$ is surjective from $X_{1}$ to $X_{1}$.

\footnotetext{
${ }^{1}$ The referee has kindly pointed out that a result of Ando [7] ensures that every isometry of $L^{p}(\Omega)(1<p<\infty, p \neq 2)$ is orthogonally complemented.
} 
COROLlaRY. If $V$ is an isometry on a smooth, reflexive Banach space which satisfies:

(1) $V$ is orthogonally complemented,

(2) $\bigcap_{n=0}^{\infty} V^{n}(X)=\{0\}$, then $V$ is a unilateral shift.

In [3] it is pointed out that for arbitrary Banach spaces it is unknown whether or not an eigenspace of an isometry has an invariant complement. However [3], this is known for invertible isometries. We have a partial solution to this problem given by:

COROLlary. Let $V$ be an isometry in a smooth, reflexive Banach space. If $V$ is orthogonally complemented then every eigenspace of $V$ has an invariant complement.

The proof follows from [3] and the previous theorem. Several questions are raised by these results. Two of these are as follows:

(1) In a Hilbert space a unilateral shift can be shown to be unitarily equivalent to an "actual" shift on $l^{2}(K)$ where $K=V(X)^{\perp}$. However, the proof does not seem to generalize in a natural fashion. Is there a similar result? If not, what operators satisfying the definitions of a unilateral shift are in some sense "natural" shifts?

(2) What isometries are not orthogonally complemented?

\section{REFERENCES}

1. G. Faulkner, Representation of linear functionals in a Banach space, Rocky Mountain J. Math. (to appear).

2. J. R. Giles, Classes of semi-inner-product spaces, Trans. Amer. Math. Soc. 129 (1967), 436-446.

3. D. Koehler and P. Rosenthal, On isometries of normed linear spaces, Studia Math. 35 (1970), 213-216.

4. J. Lamperti, On isometries of certain function spaces, Pacific J. Math. 2 (1958), 459-466.

5. G. Lumer, Semi-inner-product spaces, Trans. Amer. Math. Soc. 100 (1961), $29-43$.

6. __ On isometries of reflexive Orlicz spaces, Ann. Inst. Fourier (Grenoble), 13 (1963), 99-109.

7. T. Andô, Contractive projections in $L_{p}$ spaces, Pacific J. Math. 17 (1966), 391-405.

Department of Mathematics, North Carolina State University, Raleigh, North Carolina 27607 